\title{
A Pilot Study in Modeling Mood Disorders in Mice by Chronic Tail-Suspension Stress
}

\author{
Chun-Sheng Ruan ${ }^{1,2,+}$, Yi Guo', Larisa Bobrovskaya ${ }^{2}$, Xin-Fu Zhou ${ }^{2}$, Yue-Qin Zeng ${ }^{1,+}$
}

\begin{abstract}
Chronic stress has been known as a main cause for human mood disorders. The understanding of the pathogenesis of the mood disorders are primarily based on the usage of animal models. Low reproducibility and complicacy of production are commonly known shortcomings in the current models with chronic stress. Here, we tested a simple stress paradigm, daily exposure of 6-min tail-suspension (TS), in modelling chronic stress-induced mood disorders in mice. We found that after 2-3-weeks of TS stress mice displayed a significant decrease in exploratory behavior, increases in anxiety-like and depression-like behaviors as well as significant decreases of NeuN and GFAP levels in the hippocampus. We also found that longer exposure of mice to TS stress (up to 4 weeks) resulted in significant adaptation of these responses and reversal of most of the behavioural and biochemical changes. This study suggests that chronic TS stress for 2-3 weeks could be used as a model of mood disorders.
\end{abstract}

\section{Keywords}

Chronic stress, Tail suspension, Mood-related behavior, Hippocampus

\begin{abstract}
Introduction
A close relationship between stress and mood disorders has been well known for more than 20 years $[1,2]$ and a substantial body of evidence exists to suggest that mood disorders are frequently preceded by long-term stressful life events. Consequently, many studies have used various stress paradigms in rodents to reproduce the human condition, which include unpredictable chronic mild stress (UCMS) [3,4], chronic restraint stress (CRS) [5] and chronic social defeat stress (CSDS) [6].
\end{abstract}

Animal models based on different chronic stress paradigms have been widely used for understanding the pathogenesis or drug development of mood disorders; and low reproducibility and complex or time-consuming producing protocol are commonly seen in the current animal models. For example, the most frequently used UCMS paradigm which has been widely applied to numerous strains of rodents have over 10 different stressors and also various stress durations including 14-day $[7,8]$, 21-day [9,10] 28-day [11,12], 35- day [13], 42day $[14,15], 49-$ day $[16]$ and 56-day $[17,18]$ protocols. The diverse of UCMS protocols suggests a fact of difficulty to reproduce the model that was confirmed by our previous study, in which we found a time-dependent recovery of behavioural and biochemical changes after 8 weeks of UCMS stress [19]. Similar issues also apply to the other existing chronic stress models. In the present study, we tested a new animal model by daily exposing the mice with a 6-min tail-suspension stress for $1,2,3$ or 4 week(s), and examined the exploratory, anxiety-like and

\footnotetext{
'Yunnan Provincial Key Laboratory of Stem Cell and Regenerative Medicine, Kunming Medical University, Kunming 650500, China ${ }^{2}$ School of Pharmacy and Medical Sciences, Division of Health Sciences, University of South Australia, Adelaide, SA 5000, Australia ${ }^{\dagger}$ Author for correspondence: Chun-Sheng Ruan and Yue-Qin Zeng, Yunnan Provinical Key Laboratory of Stem Cell and Regenerative Medicine, Kunming Medical University. Email: chunsheng.ruan@mymail.unisa.edu.au (C.S.R.) and z_yueqin@hotmail.com (Y.Q.Z.)
} 
depression-like behavioural changes as well as the protein level of brain cell markers, NeuN and GFAP.

\section{Materials and Methods}

\section{- Animals}

Experiments were performed on 30 male C57BL/6 mice, 8-10 weeks old, with initial weight of 21-25 g. C57BL/6 breeders were purchased from the Vital River Laboratories (Beijing, China) and bred in the animal house of the Kunming Medical University (KMU). All breeding animals were socially-housed (no more than 5 animals per cage) with water and food freely accessed under standard housing conditions. All experimental procedures involving animals were conducted within 7 a.m. to 7 p.m., in accordance with the National Institutes of Health Guide for the Care and Use of Laboratory Animals and approved by the Department of Laboratory Animal Science of KMU. All animals were acclimatized to the housing environment for a week before being subjected to behavioural tests and stress protocols. In order to minimize the variance of baseline behaviors among groups, animals in each group were randomly and selectively grouped based on their performance of baseline behavioural tests.

\section{- Chronic tail suspension (TS) stress}

A 6-min TS [20] was daily applied as a stressor to each mouse for $1,2,3$ or 4 weeks $(n=6$ for each group, Figure 1). Meanwhile, another 6 mice were set as a control group which did not get exposed to TS stress.

\section{- Behavioural tests}

Open field test (OFT). OFT was performed as previously described [19]. Briefly, animals were placed in the center of an open arena $(50 \mathrm{~cm}$ long $\times 50 \mathrm{~cm}$ wide $\times 50 \mathrm{~cm}$ high), and allowed to move feely for $5 \mathrm{~min}$. Exploratory behaviors [20-22] as indicated by the travelling distance, turn angle of the body, rearing numbers and percentage of time spent in immobility; and anxiety-like behaviors [23,24] as indicated by decreased entries to the central zone and the percentage of time spent in the central zone, were recorded and analyzed by ANY-maze (Stoelting, Wood Dale, IL, USA) through a digital camera.

Forced swimming test (FST): FST was performed as previously described [19]. Briefly, animals were placed into a transparent Plexiglas cylinder (25 cm high; $16 \mathrm{~cm}$ diameter) containing water $\left(15 \mathrm{~cm}\right.$ high; $23-25^{\circ} \mathrm{C}$ ), and allowed to swim for $6 \mathrm{~min}$. The climbing behavior in the first 2 $\mathrm{min}$ and the immobile behavior in the last $4 \mathrm{~min}$ $[25,26]$ were recorded by a digital camera and manually analyzed. Depression-like behaviors were indicated by decreased climbing and increased immobile activities.

\section{- Sample preparation and Western blotting}

Each group of animals was humanely killed on the next day after their last behavioural testing. After the brain was perfused with ice-cold PBS, the hippocampal tissues were isolated and stored at $-80^{\circ} \mathrm{C}$. Sample preparation and Western blot were performed as previously described [19]. Briefly, tissues were homogenized in RIPA buffer (50 mM Tris- $\mathrm{HCl}, \mathrm{pH} 7.5,150 \mathrm{mM} \mathrm{NaCl}, 1 \%$ Triton X-100, 0.5\% Sodium deoxycholate and $0.1 \%$ SDS) containing protease inhibitor cocktail (Roche, Basel, Switzerland) at $4^{\circ} \mathrm{C}$ for $1 \mathrm{~h}$. The lysates were centrifuged at $12,000 \mathrm{rpm}, 4^{\circ} \mathrm{C}$ for $15 \mathrm{~min}$, and the supernatants harvested. Protein was quantified and denatured (heated at $95^{\circ} \mathrm{C}$

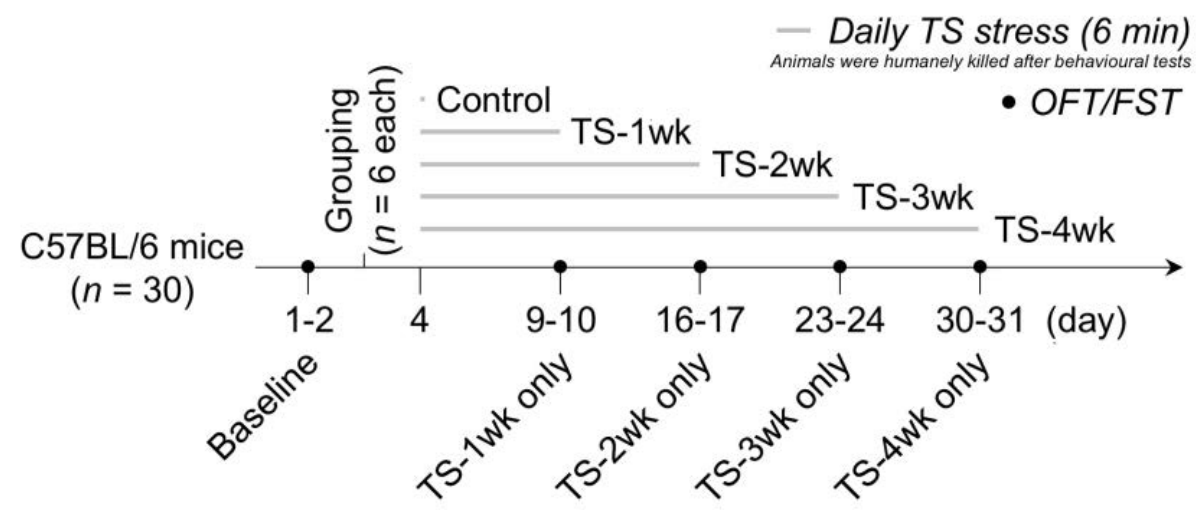

Figure 1: The time line of chronic TS stress and behavioura Itesting. TS: Tail Suspension; wk: week; OFT: Open Field Test; FST: Forced Swimming Test. 
for $5 \mathrm{~min}$ ) and the samples separated on a 10 $\%$ SDS-PAGE gel. The proteins were transferred to PVDF membrane (Millipore, Billerica, MA, USA) for $2 \mathrm{~h}$ at $400 \mathrm{~mA}$. The membranes were blocked with $5 \%$ skim milk at room temperature for $1 \mathrm{~h}$ and then incubated with mouse antiNeuN (\#MAB377; Millipore, Billerica, MA, USA) or rabbit anti-GFAP (\#ab7260; Abcam, Cambridge, UK) at $4^{\circ} \mathrm{C}$ overnight, and then incubated with HRP-conjugated secondary antibodies (CWBIO, Beijing, China) at room temperature for $1 \mathrm{~h}$. Three washes with TBST were performed after incubation with each antibody. ECL substrate (CW0049C; CWBIO, Beijing, China) was applied before imaging on ChemiDoc XRS System (Bio-Rad, Hercules, Californial, USA). Data were analyzed using Image J software (NIH, Bethesda, MD, USA).

\section{- Data analysis}

All data are presented as mean \pm standard error of the mean (SE) and analyzed by IBM SPSS Statistics 21. One-way ANOVA was used to compare the difference among groups, and leastsignificant difference (LSD) post-hoc test was used for pairwise comparison after ANOVA. $\mathrm{P}<0.05$ was considered statistically significant.

\section{Results}

- Time-dependent changes in exploratory and anxiety-like behaviors after chronic TS stress

Studies have suggested that adaptation to chronic stress can significantly affect the quality of the stress-induced animal models of mood disorders [19,27]; however, the time point when adaptation may occur is impossible to predict for each model due to numerous variables used in different studies. In this work, we employed a tail suspension (TS) stress as a physiological stressor and examined if the adaptation process will take place in C57BL/6 male animals exposed to chronic TS stress (TS for 6 min daily, as shown in Figure 1) within 4 weeks. Our data (Figure 2A, $\mathrm{P}<0.01$; Figure $2 \mathrm{~B}, \mathrm{P}<0.0001$; Figure $2 \mathrm{C}, \mathrm{P}<0.01$; Figure 2D, $\mathrm{P}<0.0001$; One-way ANOVA) showed that mice experiencing TS stress for 2 or 3 weeks showed significant decreases in the travelling distance (Figure 2A, TS-2wk, $\mathrm{P}<0.01$; TS-3wk, $\mathrm{P}<0.05$; LSD), turn angles of the body (Figure 2B, TS-2wk, $\mathrm{P}<0.0001$; TS-3wk, $\mathrm{P}<0.01$; LSD); and a significant increase in the percentage of time spent in immobility (Figure 2D, TS-2wk, $\mathrm{P}<0.0001$; TS-3wk, $\mathrm{P}<0.001 ; \mathrm{LSD})$ in OFT compared to the baseline behaviors measured before initiation of the chronic stress protocol. In addition, mice also showed a significant decrease in the rearing numbers in OFT after TS stress after 2 but not 3 weeks (Figure 2C, TS-2wk, $\mathrm{P}<0.001$; LSD). However, mice under TS stress for 4 weeks did not show significant differences in the above parameters relative to the baseline (Figures 2A-D), but showed a significant increase in the turn angles (Figure $2 \mathrm{~B}, \mathrm{P}<0.05$; LSD) and a decrease in the percentage of time spent in immobility (Figure $2 \mathrm{D}, \mathrm{P}<0.01$; LSD) compared to mice experiencing TS stress for 3 weeks. These results indicate that chronic TS stress for 2-3 weeks decreases the exploratory behavior but longer exposure to TS results in recovery of this behavior in mice. Our data (Figure 2E, $\mathrm{P}<0.05$; Figure $2 \mathrm{~F}, \mathrm{P}<0.05$; Oneway ANOVA) also showed that mice under TS stress for 2 weeks showed significant decreases in the numbers of entry to the central zone (Figure 2E, $\mathrm{P}<0.05$; LSD) and the percentage of time spent in the central zone (Figures $2 \mathrm{~F}$, $\mathrm{P}<0.05$; LSD) in OFT compared to the baseline. Moreover, mice exposed to the 3-week TS stress also showed a decrease in the percentage of time spent in the central zone (Figure 2F, $\mathrm{P}<0.05$; LSD). However, mice under TS stress for 4 weeks did not show significant differences in the above parameters relative to baseline (Figure 2EF). These results indicate that chronic TS stress for 2-3 weeks increases the anxiety-like behaviors but further exposure to TS leads to reduction of these behaviors in mice back to basal levels.

\section{- Time-dependent changes in depression-like behaviors after chronic TS stress}

FST data (Figure 3A, $\mathrm{P}<0.0001$; Figure 3B, $\quad \mathrm{P}<0.01$; One-way ANOVA) showed that mice exposed to 1-4 weeks of TS stress showed significant decreases in the percentage of time spent for climbing (Figure 3A, TS$1 w k, \mathrm{P}<0.0001$; TS-2wk, $\mathrm{P}<0.0001$; TS-3wk, $\mathrm{P}<0.0001$; TS-4wk; $\mathrm{P}<0.01$; LSD) in the first 2 minutes. Moreover, mice experiencing TS for 3-4 weeks also showed significant increases in the percentage of time spent in immobility (Figure 3B, TS-3wk, $\mathrm{P}<0.01$; TS- $4 \mathrm{wk}, \mathrm{P}<0.05)$ in the last 4 minutes. These results indicate that chronic TS stress for 3-4 weeks increases the depressionlike behaviors in mice.

- Time-dependent changes in neuronal
and glial markers after chronic TS stress

Animals from each group were humanely killed 
A
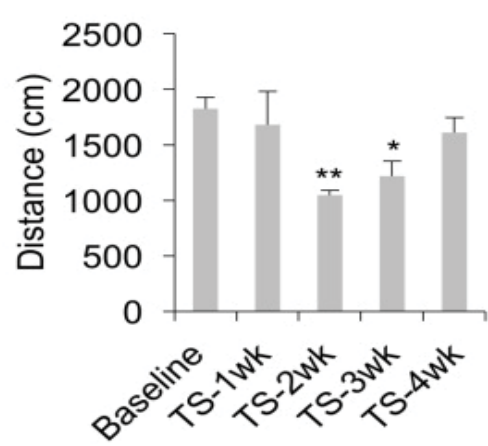

D

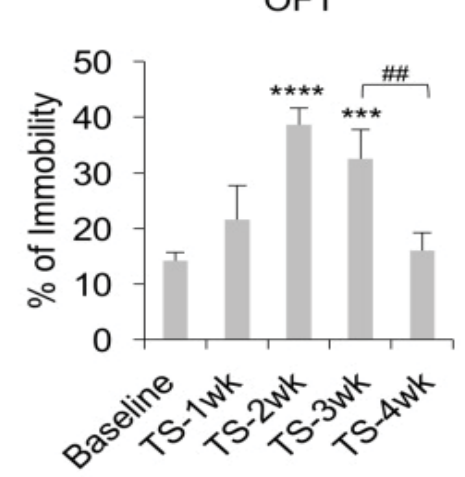

B

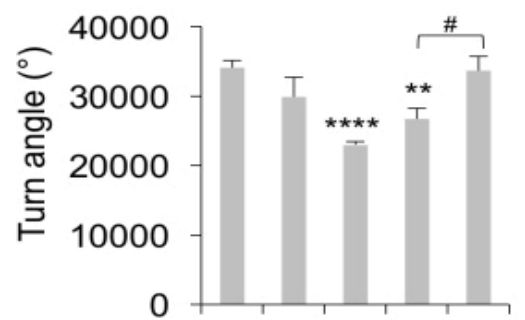

E

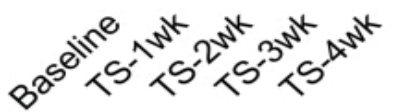

OFT

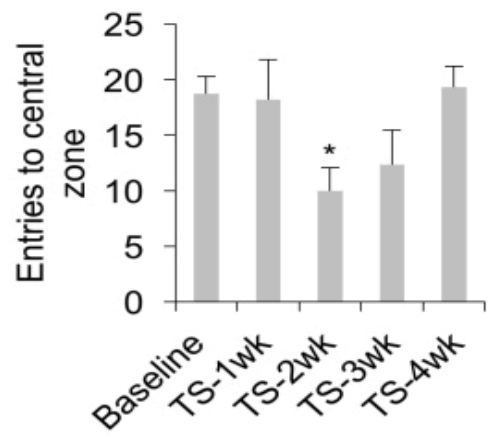

C OFT
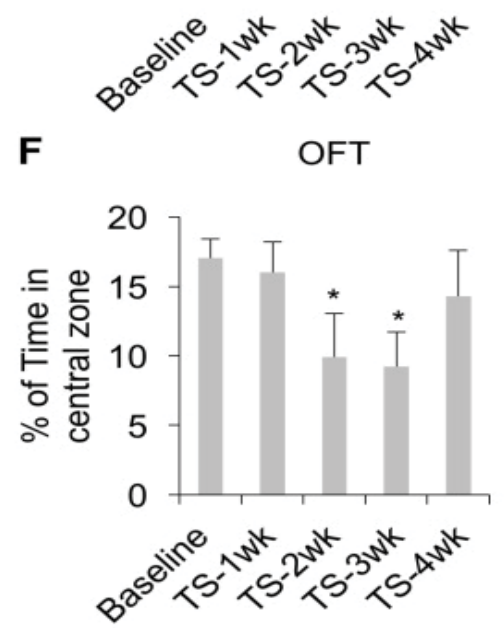

Figure 2: The time-dependent changes of exploratory behaviors and anxiety-like behaivors in OFT in mice under chronic TS stress. The travelling distance $(A)$, turn angle of body $(B)$, rearing numbers $(C)$, percentage of immobility (D), number of entries to the central zone (E) and percentage of time spent in the central zone (F) in the OFT before (baseline) and after 1, 2, 3 or 4 weeks of TS stress. TS: Tail Suspension; wk: week. ${ }^{*}, P<0.05 ;{ }^{* *}, P<0.01 ;{ }^{* * *}$, $P<0.001,{ }^{* * *}, P<0.0001$ vs baseline; $\#, P<0.05$, \#\#, $P<0.01$ vs TS-3wk (One-way ANOVA); $n=6$ each. All results are presented as mean \pm SE.

A

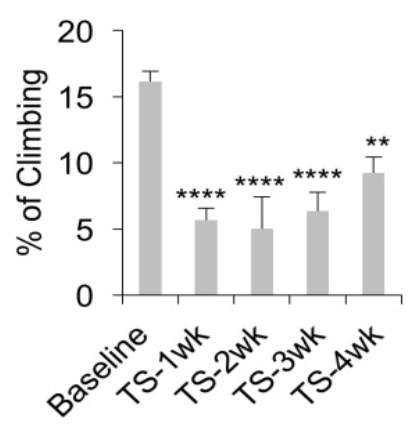

B

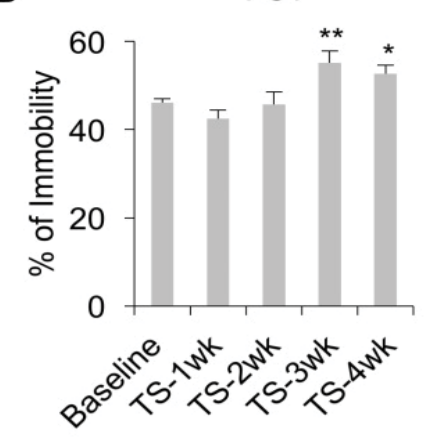

Figure 3: The time-dependent changes of depression-like behaviors in FST in mice under chronic TS stress. The percentage of time spent in climbing (A) and in immobility (B) before (baseline) and after $1,2,3$ or 4 weeks of TS stress. TS, tail suspension; wk, week; ${ }^{*}, P<0.05,{ }^{* *}, P<0.01,{ }^{* * * *}, P<0.0001$ vs baseline (One-way ANOVA); $n=6$ each. All results are presented as mean $\pm \mathrm{SE}$.

on the next day after their last behavioural tests. On completion of all experimental groups, the levels of NeuN and GFAP were detected in the hippocampal tissues by Western blot. We have found that mice under chronic TS stress for 1-4 weeks showed significant decreases in $\mathrm{NeuN}$ levels compared to the control group (Figure 4A,
$\mathrm{P}<0.0001$; One-way ANOVA; TS-1wk, $\mathrm{P}<0.05$; TS-2wk, $\mathrm{P}<0.001$; TS-3wk, $\mathrm{P}<0.001$; TS-4wk, $\mathrm{P}<0.05$; LSD); however, the decreases by week 4 were significantly smaller in comparison to those by weeks 2 and 3. Moreover, mice under TS stress for 2-3 weeks (but not 4 weeks) also showed significant decreases in GFAP levels 
A

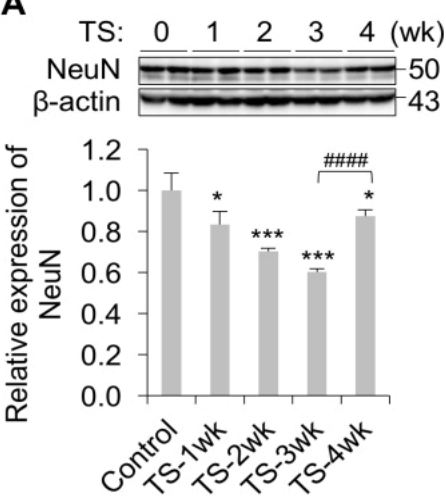

B

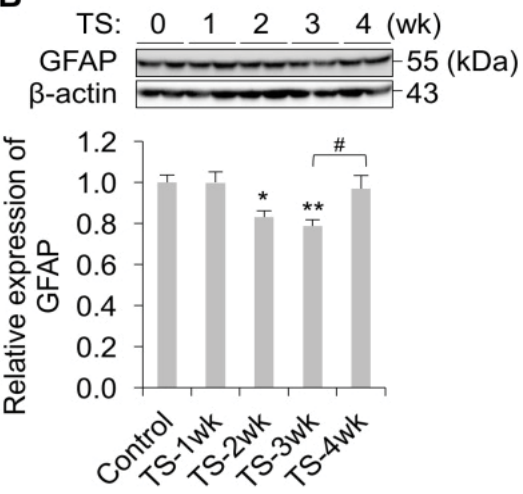

Figure 4: The time-dependent changes of NeuN and GFAP levels in the hippocampus in mice under chronic TS stress. The protein levels of NeuN (A) and GFAP (B) in the hippocampus in mice were analyzed by Western blot before (control) and after TS stress for $1,2,3$ and 4 weeks, $\beta$-actin was detected as loading control. TS, tail suspension; wk, week; ${ }^{*}, P<0.05 ;{ }^{* *}, P<0.01 ;{ }^{* * *}, P<0.001$ vs baseline; $\#, P<0.05$, \#\#\#\#, $P<0.0001$ vs TS-3wk (one-way ANOVA); $n=6$ each. All results are presented as mean $\pm \mathrm{SE}$.

relative to the control group (Figure $4 \mathrm{~B}, \mathrm{P}<0.05$; One-way ANOVA; TS-2wk, $\mathrm{P}<0.05$; TS-3wk, $\mathrm{P}<0.01$; LSD). These results indicate that chronic stress for 2-3 weeks decreases the NeuN and GFAP levels; however further exposure to TS for up to 4 weeks results in partial or full recovery (correspondingly) of their levels in mice.

\section{Discussion}

In the present study, we have investigated time-dependent changes in behaviour and neurochemical markers after chronic TS stress in mice to provide evidence that such approach can be valuable when establishing a model of mood disorders. We have found that mice exposed to TS for 2-3 weeks showed the most pronounced changes in exploratory, anxiety and depressionlike behaviour. However, further exposure to TS for up to 4 weeks showed a significant reversal of most of these changes (except for the depressionlike behaviour in FST). A similar pattern was also found for biochemical data showing that mice exposed to TS for 2-3 weeks had the most significant decreases in NeuN or GFAP levels in the hippocampus; however, mice with continuous exposure to TS for 4 weeks showed a significant reversal in the levels of these cell markers during week 4 .

Importantly, this model produced the behavioural and neurochemical outcomes similar to the previously published data in other, well established, stress-induced rodent models of mood disorders. We have found that the chronic TS stress causes negative mood-related behaviours such as decreases in exploratory behaviour and increases in anxiety-like behaviour in OFT as well as increases in depression-like behaviour in FST. These results are consistent with the previous findings in mice under different chronic stress protocols such as UCMS [12], CRS [28,29] or a combined protocol of CRS and chronic TS [20]. The present study also shows that chronic TS stress causes loss of neuronal and astrocyte markers in the hippocampus, one of the most vulnerable areas in the brain in response to stress [7]. We have found that mice under 2-3 weeks of chronic TS stress showed significant decreases in NeuN and GFAP levels; this could be due to downregulation of the corresponding proteins, the impaired hippocampal neurogenesis [30], increased cell death [31] or dysregulation of immune system [32] caused by repeated TS stress. Our findings are consistent with most of the studies using different chronic stress protocols showing similar changes in the expression of NeuN and GFAP [33-35].

The present study also shows that most of the stress-induced behavioural and biochemical alterations are diminished specifically after 3 weeks of chronic TS stress. We found that at week 4 of chronic TS stress protocol, mice showed a significant reversal of chronic TSinduced changes in exploratory and anxiety behaviors (although the depression-like behaviors were still persisting into week 4). These results suggest that mice developed adaptation to the TS stress after 3 weeks of continuous exposure to stress (especially with regards to anxiety and exploratory behaviours); however, the depressionlike behaviour was still observed at week 4 . The reason why depression-like behaviours were 
recovered much slower than exploratory and anxiety-like behaviours is not known, which suggests a different mechanism in mediating depression-like behaviours, or the damage to brain area related to this behaviours is recovered slower. Thus, longer exposure may be required if the researchers are specifically interested in the depression-like behaviours in this model. Other behavioural tests to investigate the depressionlike behaviours to confirm our results at week 4 in this model may also be required. Similarly, NeuN and GFAP levels in the hippocampus were restored significantly (partially or completely) at week 4 of the stress protocol suggesting that the adaptation phenomenon was taking place by week 4 for these specific markers. As for why the protein level of GFAP but not $\mathrm{NeuN}$ was completely reversed after 4 weeks of stress, this is likely due to the fact that the regeneration of neurons is much slower or difficult than glial cells.

To conclude, in the present study we have successfully established a new chronic stress model by daily exposing mice to TS stress for 2-3 weeks. Longer exposure to TS stress results in significant restoration of most behavioural and neurochemical changes. Future studies will be performed to further validate this model.

\section{Acknowledgements}

This work was supported by grants from the Chinese MST $2011 C B 944200$ to X.F.Z. and Major Biomedical Project in 2018 to Y.Q. Zeng. We have no conflict of interest to declare

\section{References}

1. Kessler RC. The effects of stressful life events on depression. Ann. Rev. Psychol 48(1), 191-214 (1997).

2. Kendler KS, Karkowski LM, Prescott CA Causal relationship between stressful life events and the onset of major depression. Am. J. Psychiatry 156(1), 837-841 (1999).

3. Willner P, Towell A, Sampson D, et al. Reduction of sucrose preference by chronic unpredictable mild stress, and its restoration by a tricyclic antidepressant. Psychopharmacol 93(1), 358-364 (1987).

4. Willner P. Validity, reliability and utility of the chronic mild stress model of depression: A 10-year review and evaluation. Psychopharmacol 134(1), 319-329 (1997).

5. Galea LA, Mcewen BS, Tanapat $P$, et al. Sex differences in dendritic atrophy of CA3 pyramidal neurons in response to chronic restraint stress. Neurosci 81(1), 689 (1997).

6. Keeney A, Jessop DS, Harbuz MS, et al. Differential Effects of Acute and Chronic Social Defeat Stress on Hypothalamic-Pituitary-Adrenal Axis Function and Hippocampal Serotonin Release in Mice. J. Neuroendocrinol 18(1), 330-338 (2006).

7. Molina V, Volosin M, Cancela L, et al. Effect of chronic variable stress on monoamine receptors: Influence of imipramine administration. Pharmacol. Biochem. Behav 35(1), 335-340 (1990).

8. Choi M. Son H Effects of serotonin on erythropoietin expression in mouse hippocampus. Exp. Neurobiol 22(1), 45-50 (2013).

9. Kumar B, Kuhad A, Chopra K. Neuropsychopharmacological effect of sesamol in unpredictable chronic mild stress model of depression: Behavioral and biochemical evidences. Psychopharmacol 214(1), 819-

\section{8 (2011).}

10. Reich CG, Mihalik GR, Iskander AN, et al. Adolescent chronic mild stress alters hippocampal CB1 receptor-mediated excitatory neurotransmission and plasticity. Neuroscience 253(1), 444-454 (2013).

11. Mineur YS, Belzung C, Crusio WE. Effects of unpredictable chronic mild stress on anxiety and depression-like behavior in mice. Behav. Brain. Res 175(1), 43-50 (2006).

12. Ruan CS, Zhou FH, He ZY, et al. Mice deficient for wild-type p53-induced phosphatase 1 display elevated anxiety-and depression-like behaviors. Neurosci 293(1), 12-22 (2015).

13. Liu Q, Li B, Zhu HY, et al. Clomipramine treatment reversed the glial pathology in a chronic unpredictable stress-induced rat model of depression. Eur. Neuropsychopharmacol 19(1), 796-805 (2009).

14. Mao QQ, Ip SP, Ko KM, et al. Peony glycosides produce antidepressant-like action in mice exposed to chronic unpredictable mild stress: effects on hypothalamic-pituitary-adrenal function and brain-derived neurotrophic factor. Progress. Neuro-Psychopharmacol. Biol. Psychiatry 33(1), 1211-1216 (2009).

15. Mateus-Pinheiro A, Pinto L, Bessa J, et al. Sustained remission from depressive-like behavior depends on hippocampal neurogenesis. Transl. Psychiatry 3(1), e210 (2013).

16. Laugeray A, Launay JM, Callebert J, et al. Chronic Treatment with the IDO1 Inhibitor 1-Methyl-D-Tryptophan Minimizes the Behavioural and Biochemical Abnormalities Induced by Unpredictable Chronic Mild Stress in Mice-Comparison with Fluoxetine. PloS. One 11(1), e0164337 (2016).

17. Karson A, Demirtaş T, Bayramgürler D, et al. Chronic Administration of Infliximab (TNF-a
Inhibitor) decreases depression and anxiety-like behaviour in rat model of chronic mild stress. Basic. Clin. Pharmacol. Toxicol 112(1), 335-340 (2013).

18. Stanley SC, Brooks SD, Butcher JT. Protective effect of sex on chronic stress-and depressive behavior-induced vascular dysfunction in BALB/cJ mice. J. Appl. Physiol 117(1), 959-970 (2014).

19. Ruan CS, Wang SF, Shen YJ, et al. Deletion of TRIM32 protects mice from anxiety-and depression-like behaviors under mild stress. Eur. J. Neurosci 40(1), 2680-2690 (2014).

20. Strekalova T, Spanagel R, Bartsch D. Stress-induced anhedonia in mice is associated with deficits in forced swimming and exploration. Neuropsychopharmacol 29(1), 2007-2017 (2004).

21. Walsh RN, Cummins RA. The open-field test: A critical review. Psychol. Bulletin 83(1), 482-504 (1976).

22. Paolo SD, Peana AT, Carboni V, et al. Exploratory behaviour and grooming after repeated restraint and chronic mild stress: effect of desipramine. Eur. J. Pharmacol 399(1), 43-47 (2000).

23. Belzung C, Griebel G. Measuring normal and pathological anxiety-like behaviour in mice: A review. Behav. Brain. Res 125(1), 141-149 (2001).

24. Prut L, Belzung $C$. The open field as a paradigm to measure the effects of drugs on anxiety-like behaviors: a review. Eur. J. Pharmacol 463(1), 3-33 (2003).

25. Parra A, Vinader Caerols C, Monleón S, et al. Learned immobility is also involved in the forced swimming test in mice. Psicothema 11(1), 239-246 (1999).

26. Petit-Demouliere B, Chenu F, Bourin M. Forced swimming test in mice: a review 
of antidepressant activity. Psychopharmacol 177(1), 245-255 (2005).

27. Murakami S, Imbe H, Morikawa Y, et al. Chronic stress, as well as acute stress, reduces BDNF mRNA expression in the rat hippocampus but less robustly. Neurosci. Res 53(1), 129-139 (2005).

28. Berridge CW, Dunn AJ CRF. restraint-stress decrease exploratory behavior in hypophysectomized mice. Pharmacol. Biochem. Behav 34(1), 517-519 (1989).

29. Joo Y, Choi KM, Lee YH, et al. Chronic immobilization stress induces anxiety-and depression-like behaviors and decreases transthyretin in the mouse cortex. Neurosci. letters 461(1), 121-125 (2009).

30. Dranovsky A, Hen R. Hippocampal neurogenesis: regulation by stress and antidepressants. Biol. Psychiatry 59(1), 1136-1143 (2006).

31. Mizoguchi K, Kunishita T, Chui DH, et al. Stress induces neuronal death in the hippocampus of castrated rats. Neurosci. letters 138(1), 157160 (1992).

32. Gouin JP, Hantsoo L, Kiecolt Glaser JK. Immune dysregulation and chronic stress among older adults: A review. Neuroimmunomodulation 15(1), 251-259 (2008).
33. Araya Callís $C$, Hiemke $C$, Abumaria $N$, et al. Chronic psychosocial stress and citalopram modulate the expression of the glial proteins GFAP and NDRG2 in the hippocampus. Psychopha 224(1), 209-222 (2012).

34. Orlovsky M, Dosenko V, Spiga F, et al. Hippocampus remodeling by chronic stress accompanied by GR, proteasome and caspase-3 overexpression. Brain. Res 1593(1), 83-94 (2014).

35. Han F, Xiao B, Wen L. Loss of glial cells of the hippocampus in a rat model of post-traumatic stress disorder. Neurochem. Research 40(1), 942-951 (2015). 